\title{
Analysis of Plant Bioelectric Potential Response to Illumination by Curve Fitting
}

\author{
Ki Ando*, Yuki Hasegawa ${ }^{1}$, Hidekazu Uchida ${ }^{1}$ and Akinori Kanasugi \\ Tokyo Denki University, 5 Senju-Asahi-cho, Adachi-ku, Tokyo 120-8551, Japan \\ ${ }^{1}$ Saitama University, 255 Shimo-Okubo, Sakura-ku, Saitama-shi, Saitama 338-8570, Japan
}

(Received January 27, 2014; accepted May 14, 2014)

Key words: bioelectric potential of plant, photosynthesis, photochemical reaction, carbonfixation reaction, plant factory, curve fitting

Measurement of plant bioelectric potential is one of the promising methods for evaluating plant activities. For example, the photosynthetic rate can be evaluated using the amplitude of the potential response to illumination. In our previous work, we found that this potential response consisted of two potential variations due to photochemical and carbon-fixation reactions. These reactions are important parts of the photosynthetic reaction. However, these potential variations may overlap each other. In this case, a waveform of the potential response differed variously, and it was difficult to evaluate the photosynthetic rate using its amplitude. Therefore, we performed curve fitting for estimating and separating these overlapped potential variations. First, we defined $f_{1}$ and $f_{2}$ as functions to represent the potential variations due to the photochemical and carbonfixation reactions, respectively. Additionally, we modelled the potential response to illumination as $F=f_{1}+f_{2}$ and we fit $F$ to the original data of the potential response. As a result of curve fitting, $F$ fit to the original data of the potential response very well. The amplitude of $f_{2}$ strongly correlated with the photosynthetic rate. This result suggested that information on the photosynthetic rate was recovered by curve fitting. We consider that this method can be applied to a more accurate evaluation of the photosynthetic activity using the bioelectric potential of the plant.

*Corresponding author: e-mail: kiando@mail.dendai.ac.jp 


\section{Introduction}

Recently, safe farm products have attracted considerable interest among customers. The plant factory has been developed for satisfying the demand of customers. ${ }^{(1)}$ The plant factory controls the cultivation environment and grows farm products under a closed indoor condition. ${ }^{(2)}$ This cultivation process is not affected by toxic chemicals and the weather from outside and can realize the effective cultivation of safe crops. However, the costs of equipment and controlling the environment are greater than those of conventional outdoor cultivation at the present stage. ${ }^{(3)}$ Therefore, evaluating plant activities and feeding their information back to the environment control are necessary to increase cultivation efficiency. However, there is no suitable method for practical use in the actual cultivation site. Therefore, in most cases, the plant activities are evaluated on the basis of experience and feeling of expert farmers.

For this purpose, we focused on the bioelectric potential of the plant and its application. ${ }^{(4)}$ The bioelectric potential of the plant is generated by internal ions of plant cells. It has been reported that the potential varies with the change of the cultivation environment and reflects various physiological activities of the plant. ${ }^{(5)}$ Therefore, it is expected that the physiological activities of the plant will be evaluated and monitored by measuring the bioelectric potential. For example, when the plant is illuminated, a potential response is observed. Many studies have reported that the amplitude of this potential response correlates with the photosynthetic rate of the plant. ${ }^{(6,7)}$

However, in our latest research, we reported that the amplitude of the potential response did not correlate with the photosynthetic rate depending on the conditions of the experiment such as the illumination condition. The potential response of the plant consists of multiple potential variations reflecting each physiological activity. We considered that the waveform of the potential response became different depending on the overlapping of each potential variation. In the worst case of their overlap, significant information about the potential response was lost, and we were not able to evaluate the plant activities using the amplitude of the potential response, which was one of the conventional methods. ${ }^{(8)}$

Otherwise, the integration value and frequency characteristic of the bioelectric potential are often used for the detailed analysis of the potential response..$^{(9,10)}$ These methods are effective for evaluating the resting potential and periodic response. However, the potential response to illumination, which we focused on, is a type of action potential and is observed only once for each illumination. We considered that the lost information of the overlapped potential was difficult to recover by these methods.

In this paper, we propose an analysis method that can be used to evaluate the photosynthetic rate even in this case. For this purpose, we separated the overlapped multiple potential variations and estimated each variation component by the curve fitting to the response. In particular, we prepared a composite function for modeling the overlapped multiple potential variations, and carried out the curve fitting of this function to time-series data of the bioelectric potential response. In addition, we investigated the relationship between the photosynthetic rate and the potential variation estimated by this method. 


\section{Measurement of Plant Bioelectric Potential}

\subsection{Measurement system of plant bioelectric potential}

In this experiment, we used a foliage plant called golden pothos (Epipremnun aureum) as a measuring object of the bioelectric potential. Golden pothos is tolerant to environmental changes and allows long-term stable measurement. Figure 1 shows schematically the measurement system of the plant bioelectric potential. The potential was measured using a pair of electroencephalographic disc electrodes (NE-155A, Nihon Kohden Corp.) attached to the plant surface with a conductive paste (Z-401CE, the same manufacturer). To detect the potential response induced by the photosynthetic reactions, we attached an electrode on the leaf stem as a reference electrode, and observed the potential difference using another electrode attached to the leaf surface where the photosynthesis takes place actively. The potential was measured with a digital multimeter (DMM, R6552A, ADC Corp.) and was recorded using a PC with $1 \mathrm{~s}$ sampling intervals.

The plant was placed in an airtight vessel with a volume of $22.4 \mathrm{~L}$, and the change in $\mathrm{CO}_{2}$ concentration was obtained with an NDIR-type $\mathrm{CO}_{2}$ sensor (LI-840, Meiwafosis Co., Ltd.) for evaluating the photosynthetic and respiration rates of the plant. When the plant is placed in the dark, the $\mathrm{CO}_{2}$ concentration increases owing to the respiration of the plant. We defined this $\mathrm{CO}_{2}$ variation in the airtight vessel as the respiration rate $(\mathrm{mg} /$ h). On the other hand, the $\mathrm{CO}_{2}$ concentration decreases owing to photosynthesis under illumination. However, the measured decrease in $\mathrm{CO}_{2}$ concentration consists of $\mathrm{CO}_{2}$ absorption due to photosynthesis and $\mathrm{CO}_{2}$ emission due to respiration. Therefore, we defined the sum of the respiration and measured $\mathrm{CO}_{2}$ decrease rates during illumination as the photosynthetic rate $(\mathrm{mg} / \mathrm{h})$. To accurately determine the photosynthetic rate, we used the respiration rate that was measured just before starting the illumination.

The experiment of measuring the bioelectric potential was performed in a dark room at a temperature of 25 to $35^{\circ} \mathrm{C}$ and a humidity of 60 to $70 \%$. The light source consisted

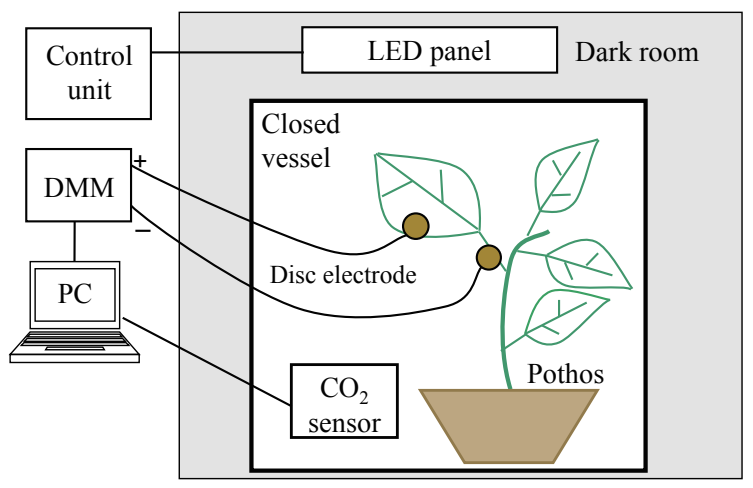

Fig. 1. (Color online) Measurement system of plant bioelectric potential. 
of blue $(470 \mathrm{~nm})$, green $(525 \mathrm{~nm})$, red $(660 \mathrm{~nm})$, and infrared $(735 \mathrm{~nm})$ LEDs (ISL305X302-RFGB, CCS), and illumination patterns were controlled with a controller (ISC101-4). In this study, the photosynthetic photon flux density (PPFD, $\mu \mathrm{mol} / \mathrm{m}^{2} \cdot \mathrm{s}$ ) was used as an index of illumination intensity and the PPFD was measured at the leaf surface. The PPFD was adjusted to $15,30,60$, and $90 \mu \mathrm{mol} / \mathrm{m}^{2} \cdot \mathrm{s}$ to control the photosynthetic rate. The PPFD ratio of LEDs was blue:green:red $=1: 1: 2$ and the color with this PPFD ratio appeared white to the eye. The PPFD was evaluated by a quantum sensor (QMSS type, Apogee). However, infrared is outside of the measurement range of the quantum sensor because infrared hardly contributes to photosynthesis. Therefore, we set the voltage of the infrared LED line to the same value as the red LED line. To inhibit the effects of attaching the electrodes and placement in the dark room, the plant was kept in total darkness for about $60 \mathrm{~min}$ prior to illuminating the LED light and recording the potential.

\subsection{Plant bioelectric potential response to illumination}

Figure 2 shows an example of the plant bioelectric potential response to starting and stopping illumination. When the illumination was started, the potential rose, dropped, and then recovered to the resting potential. The photosynthesis process of the plant can be divided into two stages. The first stage is photochemical reaction, which generates high-energy molecules by receiving light energy. The second stage is carbon-fixation reaction, which fixes carbon to phosphoric acid using the high-energy molecules. As a result of these reactions, carbohydrate was synthesized in the plant. It has been reported that the potential rise to illumination is related to the photochemical reaction and the following potential drop is related to the carbon-fixation reaction. ${ }^{(6,8,11)}$ In addition, many studies also reported that the photosynthetic rate is related to the amplitudes of these potential rise and drop as well as the amplitude of the whole response $V_{\text {on }}{ }^{(6,7)}$

On the other hand, when the illumination was stopped, the potential dropped sharply, and then recovered to the resting potential. We have reported that the intensity

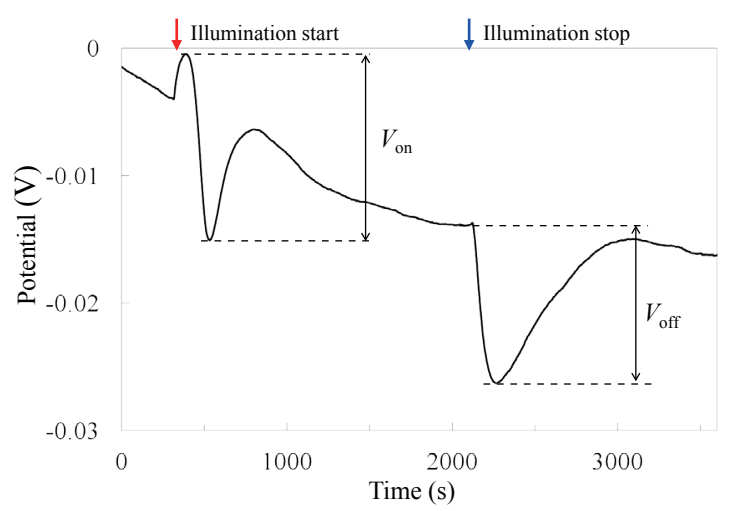

Fig. 2. (Color online) Example of plant bioelectric potential response to illumination start and stop. 
of illuminating light correlated with the amplitude $V_{\text {off }}$, and have considered that $V_{\text {off }}$ indicates the intensity of the photochemical reaction, which occurred until the illumination was stopped. ${ }^{(8)}$ The amplitudes of these responses to illumination generally range from 10 to $20 \mathrm{mV}$ under sufficient light intensity.

\section{Overlap of Potential Variations}

On the basis of our results described above, we performed further experiments. As shown in Figs. 3 and 4, we found that the potential response to illumination may take different waveforms from the response shown in Fig. 2. ${ }^{(8)}$ In the case of Fig. 3, the amplitude of the potential response was not observed clearly, and in the case of Fig. 4 , the order of the potential rise and drop was inverted. This waveform difference was observed in the experiment not only among different individuals but also with the same leaf. This tendency was obtained among three individuals and two leaves in each individual. In the case of the same leaf, the waveform differed depending on the dark period before starting illumination. We considered that the waveform was affected by

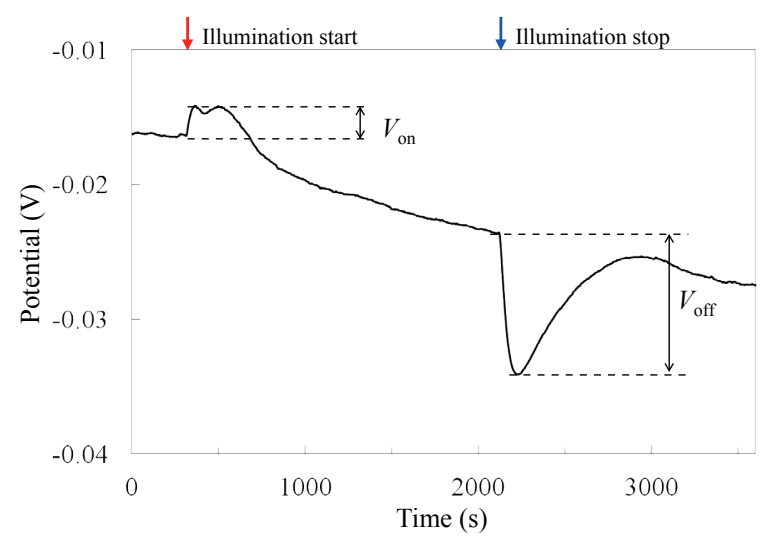

Fig. 3. (Color online) Example of unclear potential response to illumination start.
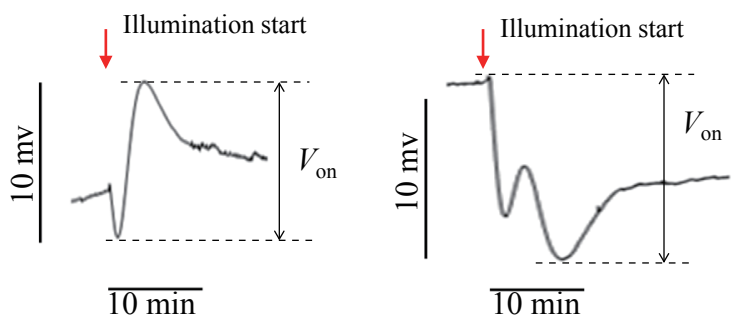

Fig. 4. (Color online) Various waveforms of potential response at illumination start. 
the carbon-fixation reaction, because the carbon-fixation reaction is deactivated slowly during a dark period. ${ }^{(8)}$

On the other hand, we found that two common peaks of the potential rise and drop can be seen in Figs. 2-4. In addition, it was considered that the waveform strongly depended on the peak time of the potential rise and drop. The peak times of these responses, as well as their amplitudes, also differ from those of the photosynthetic activities. However, when these peak times were close to each other, the potential rise and drop overlapped and information on the amplitude was lost.

When the potential responses took various waveforms and overlapped significantly, we were not able to evaluate the photosynthetic rate using their amplitudes directly. Figure 5 shows the relationship between the photosynthetic rate and the amplitude $V_{\text {on }}$. In this experiment, the potential was measured from the same individual and the same leaf. The dark period before illumination start was 30 or 60 min and the illumination intensity differed in each measurement. We obtained $V_{\text {on }}$ by subtracting the value just before starting illumination from the peak value of the potential rise. The potential responses took various waveforms, as shown in Figs. 2-4, depending on the dark period. As a result, $V_{\text {on }}$ showed no correlation with the photosynthetic rate. From this result, it was considered that the photosynthetic rate may be difficult to evaluate by simply using the amplitude of the potential response.

\section{Analyzing Potential Response by Curve Fitting}

\subsection{Definition of fitting function}

As described above, two potential variations, the potential rise and drop, were mainly observed in the potential response to illumination. Additionally, it was considered that these potential variations were due to the photochemical and carbon-fixation reactions in the photosynthetic process, respectively. We expected that if these potential rise and drop

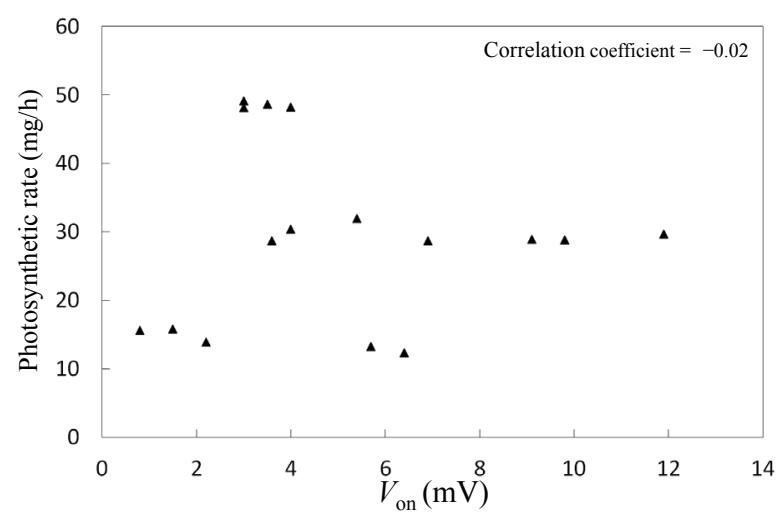

Fig. 5. Relationship between amplitude $V_{\text {on }}$ obtained from the responses of various waveforms and photosynthetic rate. 
were separated from the overlapped potential response, any waveform of the response could be used for evaluating the photosynthetic activities. Therefore, we performed curve fitting to estimate the potential rise and drop.

First, we defined $f_{1}$ and $f_{2}$ as functions to represent the potential rise and drop, respectively. $f_{1}$ and $f_{2}$ were functions of time $t$ determined by modifying the Gumbel distribution. The original Gumbel distribution is a type of probability density function shown as

$$
f(x)=\frac{1}{\eta} \exp \left\{-\exp \left(-\frac{x-\mu}{\eta}\right)-\frac{x-\mu}{\eta}\right\}
$$

The Gumbel distribution is a left-right asymmetry function, and the slope of the right side is gentler than that of the left side under $\eta>0$. In addition, $\mu$ is related to the position of the peak and $\eta$ is related to the peak value of the function. We thought that this characteristic is similar to the behavior of the bioelectric potential. However, the Gumbel distribution has no independent coefficient related to the degrees of the slopes. Therefore, we modified the Gumbel distribution as shown in eq. (2) and used this function for curve fitting.

$$
f(t)=p_{1} \exp \left\{-\exp \left(-\frac{t-p_{3}}{p_{2}}\right)-\frac{t-p_{5}}{p_{4}}+1\right\}
$$

The number of coefficients was increased to 5. Each coefficient is mainly related to the characteristics of the function as follows. $p_{1}$ and $p_{5}$ are related to the peak value of the function. $p_{2}$ and $p_{4}$ are related to the degrees of the slopes before and after the peak, respectively. $p_{3}$ is related to the peak time of the function. This formula can be transformed more simply. However, we used this expression because the contribution of each coefficient could easily be understood.

Then, we defined $F$ as $F=f_{1}+f_{2} . \quad F$ was the modelization of the whole potential response induced by illumination. We obtained the coefficients $p_{1}$ to $p_{5}$ of $f_{1}$ and $f_{2}$ to fit $F$ to the original time-series data of the plant bioelectric potential. This calculation was carried out using a solver of Microsoft Excel by the least-squares approach.

In addition, we made two corrections to the time-series data before performing curve fitting to obtain a better fitting result. First, the bioelectric potential often has a gentle slope, as shown in Figs. 2 and 3. It has been reported that this slope is related to the time of day and watering condition. ${ }^{(12)}$ However, this slope was not considered in this study, and the modelling function $F$ did not have a member corresponding to the slope. Therefore, we corrected the slope of the potential response to become a horizontal slope as a whole. Second, the data obtained before starting and after stopping the illumination were not modelled in $F$, and these parts interfered with obtaining a fine fitting result. Therefore, we used the time-series data between the beginning of illumination and the recovery to the resting potential. 
The Gumbel distribution used in this study is an example of functions for the curve fitting to the potential response. We think that there may be a more suitable function for this purpose.

\subsection{Estimation of overlapped potential variations}

Figure 6 shows an example of performing the curve fitting to the potential response to illumination shown in Fig. 2. In Fig. 6, the solid line shows the original data of the potential response, and the broken line shows the result of the curve fitting $F$. The broken line represents almost the same waveform as the solid line. This finding suggests that the result of the curve fitting is good. $f_{1}$ and $f_{2}$, which constitute $F$, are represented by the gray solid and dotted lines in Fig. 6, respectively. We defined the amplitudes of $f_{1}$ and $f_{2}$ as $V_{f 1}$ and $V_{f 2}$, respectively.

Figure 7 shows the result of the curve fitting to the response shown in Fig. 3. In contrast to Fig. 2, it seemed that the potential rise and drop of the target response overlapped deeply, and their amplitudes were small and unclear. However, the waveform of $F$ fit the original data very well, and $f_{1}$ and $f_{2}$ obtained using the curve fitting represented a similar waveform to the result shown in Fig. 6. Moreover, $V_{f 1}$ and $V_{f 2}$ were larger than the amplitude of the original data.

In addition to these results, we performed the curve fitting to various waveforms of the potential response such as those shown in Figs. 4(a) and 4(b). In most results of the curve fitting, the waveform of $F$ was able to fit to the original data of the potential response. We considered that the actual potential rise $f_{1}$ and actual potential $\operatorname{drop} f_{2}$ were estimated and separated from the potential response to illumination by curve fitting. Investigation of the relationship between this estimation result and the photosynthetic rate was explained in $\S 5$.
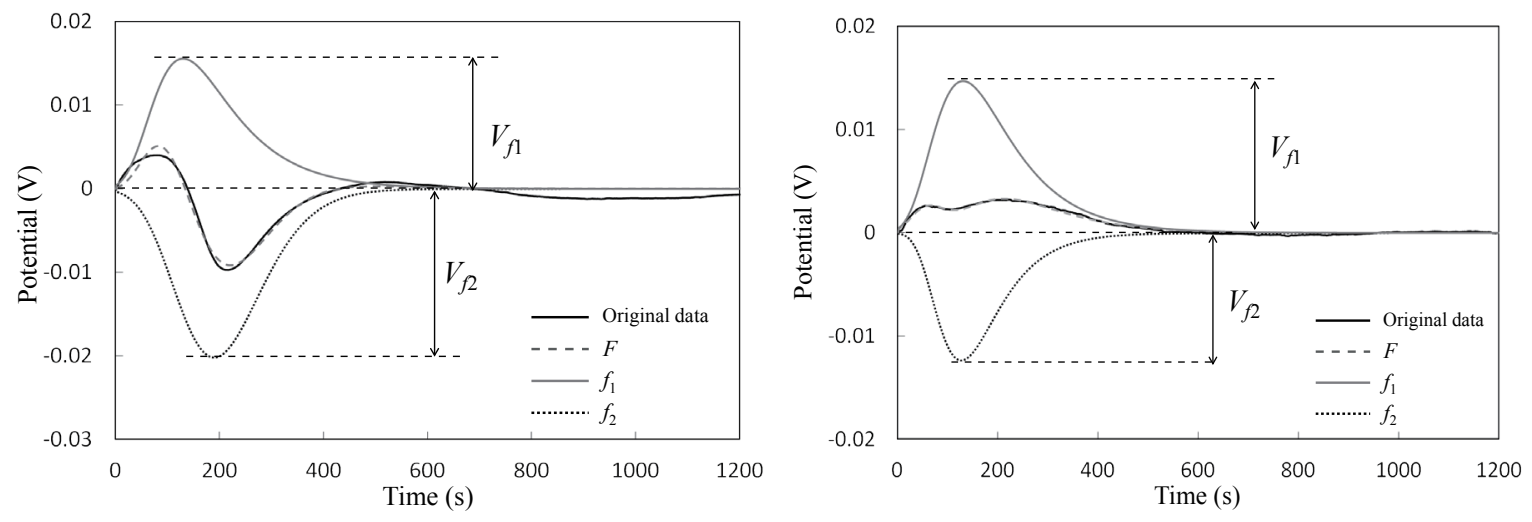

Fig. 6 (left). Result of curve fitting to the response with clear amplitude.

Fig. 7 (right). Result of curve fitting to the response with unclear amplitude. 


\subsection{Restriction condition to curve fitting}

In the case of some potential response, even though the waveform of $F$ fit to the original data, $f_{1}$ and $f_{2}$ may take multiple combinations. Figure 8 shows an example of a different combination of $f_{1}$ and $f_{2}$ obtained from the same original data. In the comparison of (a) and (b), the result of the curve fitting $F$ and the residual sum obtained by the least-squares approach were almost the same. However, the amplitudes of $f_{1}$ and $f_{2}$ differed significantly between (a) and (b). We considered that this problem was caused by a very wide range of coefficients that $f_{1}$ and $f_{2}$ could take.

To avoid this problem, we appended a restriction to the curve fitting, which was $V_{f 1}$ $\approx V_{\text {off }} . V_{\text {off }}$ indicated the amplitude of the potential response at stopping illumination, as shown in Figs. 2 and 3. The basis of this restriction is shown as follows.

First, Fig. 9 shows the potential response induced by illumination under the $\mathrm{N}_{2}$ gas condition, which we reported previously. ${ }^{(8,11)}$ In $\mathrm{N}_{2}$ gas, the carbon-fixation reaction is inhibited owing to the $\mathrm{CO}_{2}$-free condition. In addition, both amplitudes, $V_{\text {on-N2 }}$ and $V_{\text {off-N2 }}$, correlated with the intensity of illumination. Therefore, we considered that these responses were commonly due to the photochemical reaction. In particular, the amplitude $V_{\text {on-N2 }}$ usually indicated a similar value to $V_{\text {off-N2 }}$ without depending on the experimental condition and their correlation coefficient was $0.97 .^{(11)}$

On the other hand, as described in $\S \S 2.2$ and 4.1, the potential rise at illumination $\left(f_{1}\right)$ and the potential response at stopping illumination were also related to the photochemical reaction. Therefore, we assumed that $V_{f 1}$ indicated the same amplitude as $V_{\text {off }}$ as well as the relationship between $V_{\text {on-N2 }}$ and $V_{\text {off-N2 }}$ in $\mathrm{N}_{2}$ gas. In the experiment, we obtained $V_{\text {off }}$ by subtracting the peak value of the potential drop from the value just before stopping illumination. This value was used for the restriction condition of the curve fitting.
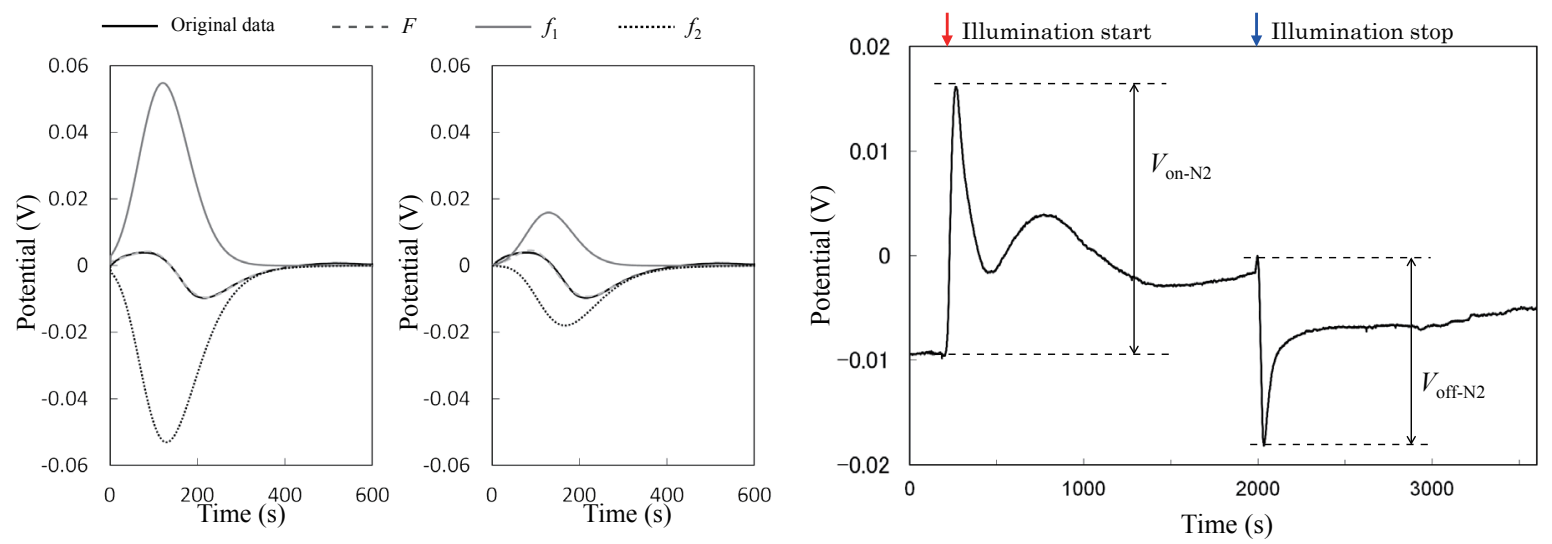

Fig. 8 (left). Different results of curve fitting obtained from the same original data.

Fig. 9 (right). (Color online) Bioelectric potential response to illumination in $\mathrm{N}_{2}$ gas for inhibiting carbon-fixation reaction. ${ }^{(11)}$ 


\section{Relationship between Estimation Result and Photosynthetic Rate}

\subsection{Amplitude of potential drop $\mathrm{V}_{\mathrm{f} 2}$ and photosynthetic rate}

In this section, we investigated the relationship between the estimated amplitude of the actual potential drop $V_{f 2}$ and the photosynthetic rate. As described in Fig. 3, it is considered that the potential drop response to illumination is due to the carbon-fixation reaction. ${ }^{(6,8,11)}$ Therefore, we predicted that if the potential drop $f_{2}$ were estimated correctly by curve fitting, its amplitude $V_{f 2}$ would strongly correlate with the intensity of the carbon-fixation reaction, namely, the photosynthetic rate.

Figure 10 shows the relationship between the estimated amplitude of the potential drop $V_{f 2}$ and the photosynthetic rate. The original data for the curve fitting is completely the same as the data used in Fig. 5. Although $V_{f_{2}}$ had a certain variance with each photosynthetic rate range, it was approximately proportional to the photosynthetic rate. The correlation coefficient was about 0.85 . In comparison with the result shown in Fig. 5 using unprocessed data, the variance of the amplitude became smaller, and a proportional relationship was observed.

From this result, we considered that the information lost by overlapping the potential rise and drop could be estimated and recovered by curve fitting, and that the photosynthetic rate could be evaluated using the estimated amplitude $V_{f 2}$.

\subsection{Restriction condition $\mathrm{V}_{\text {off }}$ and photosynthetic rate}

In the previous section, the amplitude $V_{f 2}$ correlated with the photosynthetic rate. However, this $V_{f 2}$ was obtained under the restriction condition $V_{f 1}=V_{\text {off }}$ in the curve fitting, as described in $\S 4.3$. We considered that the result shown in Fig. 10 could possibly indicate the relationship between $V_{\text {off }}$ and the photosynthetic rate. Therefore, we also investigated this relationship and showed the result in Fig. 11. The amplitude $V_{\text {off }}$ was obtained from the same original data shown in Figs. 5 and 10. The correlation coefficient was 0.67 , and the photosynthetic rate was observed to be proportionally

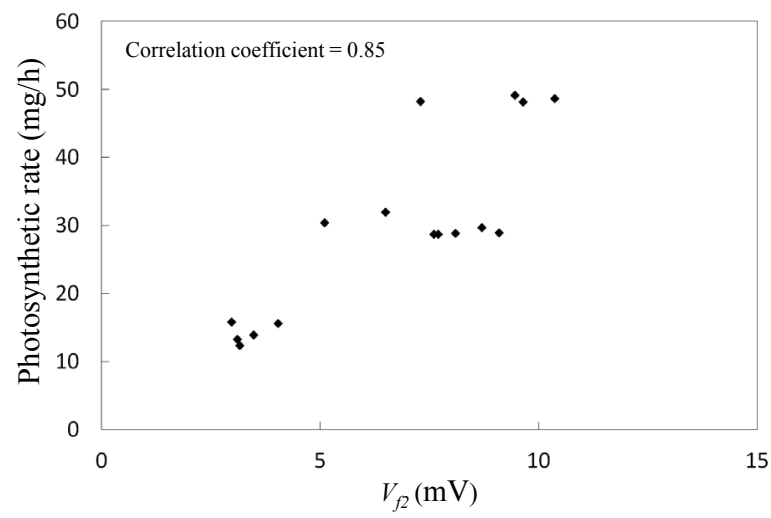

Fig. 10. Relationship between estimated amplitude $V_{f 2}$ and photosynthetic rate. 


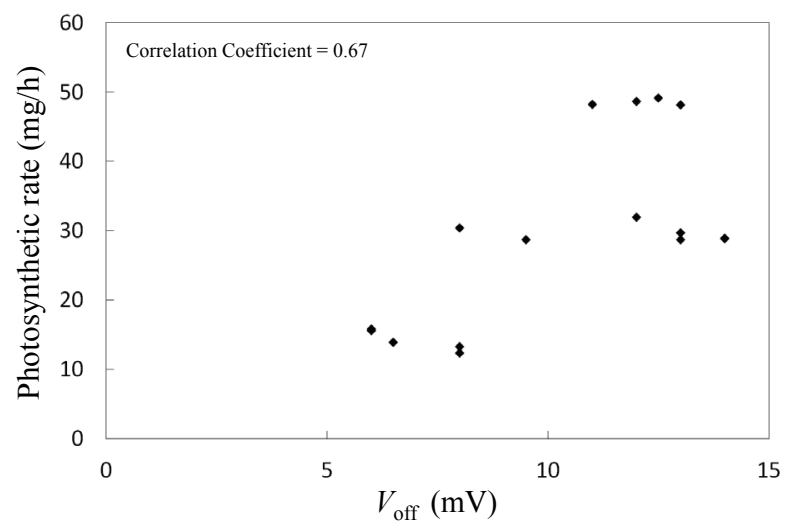

Fig. 11. Relationship between amplitude $V_{\text {off }}$ used for restriction condition and photosynthetic rate.

related to $V_{\text {off }}$. However, the correlation coefficient of $V_{f 2}$ (Fig. 10) was greater than that of $V_{\text {off }}$ (Fig. 11).

Therefore, $V_{f 2}$ was considered to be more strongly related to the carbon-fixation reaction than $V_{\text {off. }}$. In other words, the correlation with the photosynthetic rate was improved by estimating $V_{f 2}$ using $V_{\text {off. }}$ In comparison with the result obtained using raw amplitude data (Fig. 5), the result in Fig. 10 suggested a significant recovery of the lost information on the carbon-fixation reaction. We considered that this curve fitting can be applied to a more accurate evaluation of the photosynthetic rate.

\section{Conclusions}

The plant bioelectric potential response induced by illumination consisted of the potential rise due to the photochemical reaction and the potential drop due to the carbonfixation reaction. When these potential variations overlapped deeply, the information on the potential amplitude related to the photosynthetic activities was lost. In this case, we could not evaluate the photosynthetic rate using the potential amplitude. Therefore, we performed the curve fitting to the potential response to estimate and separate these overlapped potential variations.

For this purpose, we defined the potential rise and drop as two Gumbel distribution functions, and modelled the whole potential response to illumination using the sum of two Gumbel distribution functions. The Gumbel distribution used in this study is an example of functions for the curve fitting to the plant bioelectric potential response. We think that there may be a more suitable function for this purpose. Additionally, we appended the restriction condition to the curve fitting for obtaining better results.

As a result of the curve fitting, the waveform of the modelled function fit the original data very well, and the potential rise and drop were estimated and separated. The estimated potential drop correlated with the photosynthetic rate strongly. This result 
suggested that the lost information on the carbon-fixation reaction was recovered. We considered that this curve fitting can be applied to a more accurate evaluation of the photosynthetic rate.

\section{References}

1 M. Takatsuji: Fundamentals and Practices of Plant Factories (Shokabo, Tokyo, 2007) (in Japanese).

2 M. Takatsuji: J. Soc. High Technol. Agric. 22 (2010) 2 (in Japanese).

3 M. Nishimura, T. Kozai, C. Kubota and C. Chun: J. Soc. High Technol. Agric. 13 (2001) 204 (in Japanese).

4 M. Tazawa, T. Hhinmen and T. Mimura: J. Inst. Electrostat. Jpn. 6 (1982) 285 (in Japanese).

5 T. Hirao and N. Arai: Bull. Natl. Inst. Agrobiol. Resour. 4 (1988) 65 (in Japanese).

6 K. Harada: T. IEE Japan 119-E (1999) 270 (in Japanese).

7 K. Ando, Y. Hasegawa, H. Maekawa and T. Katsube: IEICE Trans. Electron. E91-C (2008) 1905.

8 K. Ando, Y. Hasegawa, T. Yaji and H. Uchida: Electron. Comm. Jpn. 96 (2013) 85.

9 S. Shibata, T. Oyabu and H. Kimura: 40th Int. Conf. on Computers and Industrial Engineering (2010).

10 S. Shibata, F. Satou, H. Kimura and T. Oyabu: IEEJ Trans. SM. 132 (2012) 154 (in Japanese).

11 K. Ando, Y. Hasegawa, T. Yaji and H. Uchida: Electron. Comm. Jpn. 95 (2012) 10.

12 K. Nakamura and M. Fujimoto: IEICE Trans. Inf. \& Syst. J81-D-2 (1998) 769 (in Japanese). 